\title{
LVRT Impact on Tower Loads, Drivetrain Torque and Rotational Speed-Measurement Results of a 2-MW Class DFIG Wind Turbine
}

\author{
Mathias Arbeiter ${ }^{1}$, Martin Hopp ${ }^{1}$ and Martin Huhn ${ }^{2, *}$ \\ 1 Suzlon Energy Ltd., German Branch, 18057 Rostock, Germany; mathias.arbeiter@suzlon.com (M.A.); \\ martin.hopp@suzlon.com (M.H.) \\ 2 Department of Mechanical Engineering and Business Administration, Technical University of Applied \\ Sciences Lübeck, 23562 Lübeck, Germany \\ * Correspondence: martin.huhn@th-luebeck.de
}

Citation: Arbeiter, M.; Hopp, M.; Huhn, M. LVRT Impact on Tower Loads, Drivetrain Torque and Rotational Speed-Measurement Results of a 2-MW Class DFIG Wind Turbine. Energies 2021, 14, 3539. https://doi.org/10.3390/en14123539

Academic Editors: Frede Blaabjerg and Mohamed Benbouzid

Received: 12 March 2021

Accepted: 3 June 2021

Published: 14 June 2021

Publisher's Note: MDPI stays neutral with regard to jurisdictional claims in published maps and institutional affiliations.

Copyright: (C) 2021 by the authors. Licensee MDPI, Basel, Switzerland. This article is an open access article distributed under the terms and conditions of the Creative Commons Attribution (CC BY) license (https:// creativecommons.org/licenses/by/ $4.0 /)$.

\begin{abstract}
With an increasing share in energy production, wind turbines have to fulfill strict grid requirements to support the grid in case of discontinuities. Here LVRT (Low Voltage Ride Through) compliance is one essential part, where the turbine has to stay connected to the grid in case of voltage drops and is not allowed to stop. This paper comprises measured low-voltage events from an LVRT campaign with a focus on the mechanical loads for the drivetrain and tower during these temporary and instant drops of power. Moreover, we analyze the turbine operation itself, like the rotational speed, which is essential to keep the turbine within its operational parameters and design limits.
\end{abstract}

Keywords: wind turbine; low voltage ride through; LVRT; fault ride through (FRT); grid code; grid compliance; voltage dip depth; power recovery time; measurement; loads; turbine operation; over speed; tower oscillation; drivetrain eigenfrequency; drivetrain torque; tower loads

\section{Introduction}

Renewable energies play a central part in the transition to more sustainable energy production. In the future, it is expected that their contribution will further increase to a significant extent. This leads to the need for higher efforts for renewable energy to contribute to grid stability and to fulfill modern grid requirements.

Here LVRT compliance plays a central role. In case of sudden voltage drops, the wind turbines must stay connected to the grid and are forced to continue with power production once the grid has recovered.

Over the last years, the LVRT requirements have become more demanding in terms of voltage dip depth and duration, and even voltage drops to zero have become part of the requirement for some grid codes. In addition, stricter requirements for the power recovery further push the turbines towards their limit of operation, e.g., for the India grid code CEA 2019 [1] a power return to $90 \%$ is required within one second after voltage comeback.

This trend of more challenging LVRT scenarios is potentially a design driver for the turbines. Thus, LVRT events become part of the design load cases for certification simulation in some guidelines, e.g., the IEC Guideline Edition 4 [2].

Even if the share of wind turbines with doubly-fed induction generators (DFIG) in new installations is decreasing, the amount was $17.9 \%$ in 2018 [3]. DFIG is still widespread in countries such as China and India [4-6].

This requirement led in the last years to the development of several technical solutions to operate various types of wind turbines during LVRT [7], and for DFIG based wind turbines, specifically [8,9]. Special attention has been put on DFIG due to its high sensitivity for grid voltage faults, e.g., the implementation of a control algorithm for rotor and grid side converters [10], the usage of fault current limiting devices [11], and investigations of 
protection elements for the electric components like a crowbar or stator current feedback solution [12].

Since the main focus of many publications is on simulations, this publication contains measurement results of mechanical loads and the turbine operation itself, including known risks like over-speed and excitation of the drivetrain eigenfrequency of the turbine [13-15].

The following two main concerns are addressed in this paper:

- Can LVRT events lead to extreme loads in key components like tower and drivetrain, potentially harming the integrity of the turbine?

- Can LVRT events interfere with the turbine operation in a way that its boundary conditions such as safety routines and rotational speed-limited components are exceeded leading immediately to disconnection and stoppage of the turbine?

For more insight into these basic questions, we present measurement data of wind turbines for various LVRT scenarios as well as numerical simulations with Flex5 [16] for more detailed parameter studies where measurement trials are limited.

The basis for the measured signals is an LVRT campaign, which has been performed on a turbine of the manufacturer Suzlon in the $2 \mathrm{MW}$ class with rotor diameters larger than $100 \mathrm{~m}$ and a speed limited DFIG. The DFIG includes a DC-link chopper that is active during an LVRT and prevents overcurrent and overvoltage. Moreover, a crowbar is installed as an additional safety element in case of malfunction to protect the converter. During an LVRT event, the control system of the converter maintains a power output that is proportional to the voltage drop at the time. Power return after voltage comeback can be parametrized with appropriate ramp rates. More information about DFIG can be found in [17].

The turbine is equipped with strain gauges at the high-speed shaft of the drivetrain as well as on the tower bottom. In addition, acceleration sensors at the mainframe monitor the oscillations of the tower top.

For the evaluation of the mechanical loads and the turbine operation we focus on the following items:

1. acceleration of rotor speed and imminent over-speed due to drivetrain oscillations

2. drivetrain torque loads at the High-speed-shaft (HSS)

3. tower bending loads and tower top oscillations

These three issues appear as most sensitive and critical for the turbine during and right after LVRT events. We will quantify the impact of LVRTs on the mechanical loads and the turbine operation in dependency of the LVRT parameter, i.e., the duration and voltage dip depth.

The structure of this publication is as follows: In Section 2, we will give background information about the measurement campaign itself and then present final results for rotor speed, drivetrain torque, and tower oscillations in a summarized way for the whole set of LVRT scenarios. The measured data are further analyzed and discussed in individual Sections $3-5$ for each of the three items including simulation results to support and explain the observations.

The results shown in this publication demonstrate the feasibility of DFIG based wind turbines to cope with modern grid codes and in parallel point to the most critical elements in turbine design which may become the bottleneck for compliance to upcoming grid codes.

\section{LVRT Events of a Measurement Campaign-Impact on Critical Values}

In this section, we show exemplary temporal evolution during an LVRT event of some key sensors which are in the focus for the entire paper. This will guide to the critical parts of turbine loads and operation which are then discussed individually in more detail in the subsequent sections.

Afterward, the results are presented for a bunch of different LVRT scenarios which have been part of an entire LVRT campaign. 
At first, the main characteristics of an LVRT event are clarified. Since the focus of investigations is on the mechanical structure of the turbine and the turbine operation itself, the following terms are of most interest for an LVRT event:

- $\quad$ LVRT dip depth refers to the remaining voltage level during an LVRT event, i.e., a $0 \%$ LVRT dip depth refers to a temporary grid loss event. In the entire publication, we are focusing on the simultaneous voltage drop of all three phases.

- $\quad$ LVRT duration refers to the duration of reduced voltage levels. In this paper, we are focusing on LVRT events with a rectangular shape of voltage evolution, i.e., a sharp voltage drop to a certain level, a constant reduced level of voltage for the time of the LVRT duration, and finally a sudden voltage comeback. Other temporal evolutions of the voltage drop may occur in reality but are seen as less critical from the loads' point of view. The measured LVRT events presented in this paper are induced artificially by LVRT test equipment leading to sharp voltage drops and sharp voltage returns.

- LVRT power recovery time determines the time from the voltage comeback, i.e., the end of LVRT, to the point where the desired power level is reached. Modern grid codes require a quick power recovery time, e.g., the India grid code CEA 2019 requires achieving $90 \%$ of the pre-LVRT power level within one second.

Other LVRT parameters like reactive power and the individual voltage levels of the three current phases, which have to be considered to ensure the operation of the electrical components, are not considered in this publication.

Figure 1 depicts a measured LVRT example with a voltage dip depth of approximately $30 \%$ and a duration of $1.1 \mathrm{~s}$. The values are normalized to the pre-LVRT situation, except for the pitch angle given in degrees and for the tower top acceleration where the absolute root mean square values are given.

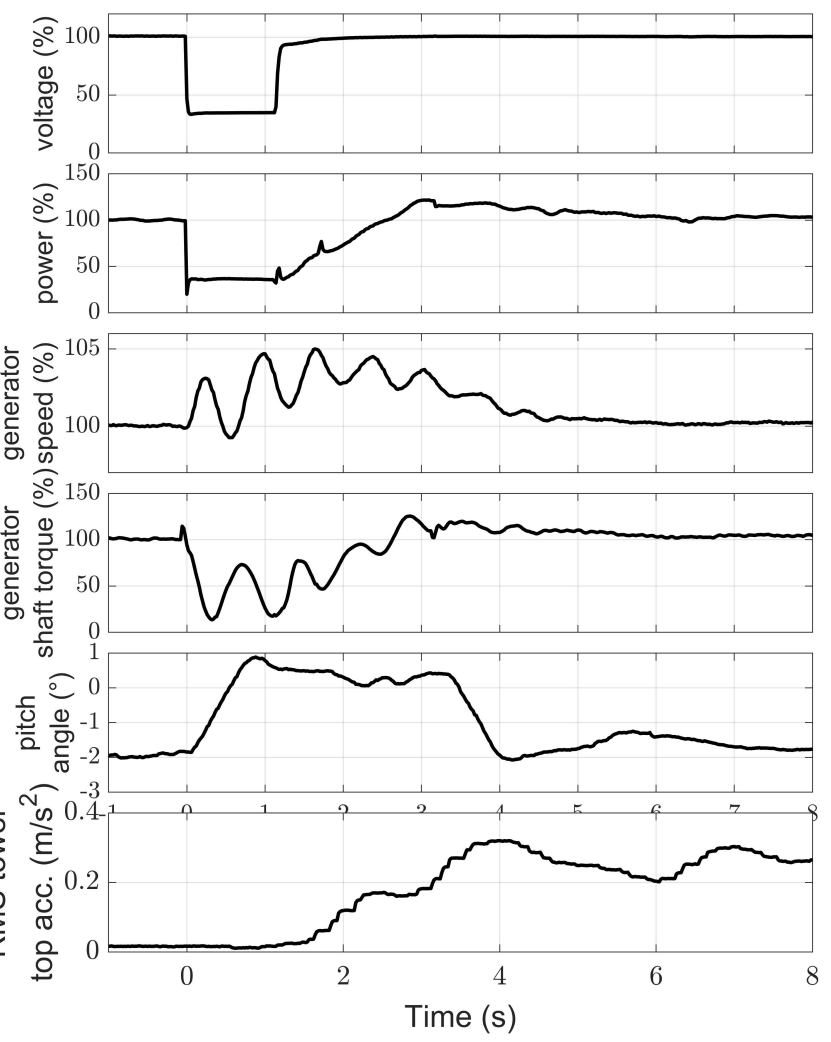

Figure 1. Temporal evolution of voltage, electrical power, generator speed, drivetrain torque, pitch angle and tower top acceleration for a measured LVRT with $30 \%$ voltage drop and duration of $1.1 \mathrm{~s}$. For tower top acceleration the corresponding RMS value is shown. All other signals are normalized to their values right before the LVRT except for the pitch angle given in degree. 
The voltage dip is preset with an instantaneous drop, a constant plateau at $30 \%$, and an instantaneous return of voltage. The voltage dip leads directly to a sudden drop of power at $\mathrm{t}=0 \mathrm{~s}$ roughly proportional to the voltage drop. With this decrease of electrical generator power, the turbine quickly speeds-up, since the pitch system is not capable of fully counteracting the sudden speed increase on such short timescales.

Moreover, the sudden loss of electrical power and consequently generator torque induce a strong drivetrain oscillation, which is visible in the generator speed itself as well as visible in the torque signal. The oscillating generator speed already exceeds significantly the initial speed level even before the voltage returns. Here the oscillation frequency is in the order of the eigenfrequency of the drivetrain.

Even the fast responses of pitch drives with a pitch speed of $4^{\circ} / \mathrm{s}$ used in the shown scenario are not sufficient to fully counteract the rotor acceleration. Higher pitch speeds would slightly improve the situation but especially for the deep and short LVRTs below $20 \%$ voltage dip and durations less than one second, the pitch effect is limited on these short timescales.

In Section 3, the acceleration of speed will be discussed in more detail, whereas in Section 4 the torque of the generator shaft is under detailed consideration.

On a larger timescale, the tower gets excited as well, where the oscillation can persist for 20-40 s due to the comparatively low damping. Especially in the fore-aft direction, the tower accelerates due to the sudden drop of torque and the subsequent pitch in and out activity to control the turbine speed, see the tower top acceleration in the bottom panel of Figure 1. Section 5 focuses on these accelerations and the corresponding tower bottom bending moments.

All three signals, namely, generator speed, drivetrain torque, and tower moments play an important role in the turbine design for the loads themselves and for the proper turbine behavior within its specified range and are therefore in the focus of LVRT measurement campaigns.

In the following, we present various LVRT measurements with different LVRT parameter settings performed at one 2-MW-class wind turbine. The results are displayed in Figure 2 where the maximum observed values for the generator speed (Figure 2a), the drivetrain torque at the high-speed shaft (Figure 2b), and the maximum RMS tower top acceleration (Figure 2c) are given.

For the generator speed and the drivetrain torque, the maximum values are given in percentage relative to the expected values above rated wind speed. For tower top acceleration the absolute maximum RMS values are shown.

Open circles reflect the pre-LVRT value right before the voltage drop and the filled circles correspond to the maximum observed values during and after the LVRT event. The color code represents the four parameter sets of LVRT scenarios as described in the legend. The chosen parameter sets reflect the grid requirement that the turbine has to stay connected for longer times in case of less deep voltage dip depths.

For the generator speed (Figure $2 \mathrm{a}$ ), maximum values of almost $10 \%$ above the rated speed have been measured. Moreover, higher dip depths appear as more critical for the turbine compared to longer but less deep LVRTs. More detailed analyses are given in Section 3.

The drivetrain torque (Figure $2 \mathrm{~b}$ ) shows even an increase of $40 \%$ where the $0 \%$ LVRT is clearly the most critical scenario. This is further discussed and analyzed in Section 4.

Even for the tower top acceleration (Figure 2c), there is a clear impact from the LVRT event visible. The tower shakes considerably harder with increasing voltage dip depth, see Section 5 for more details. 

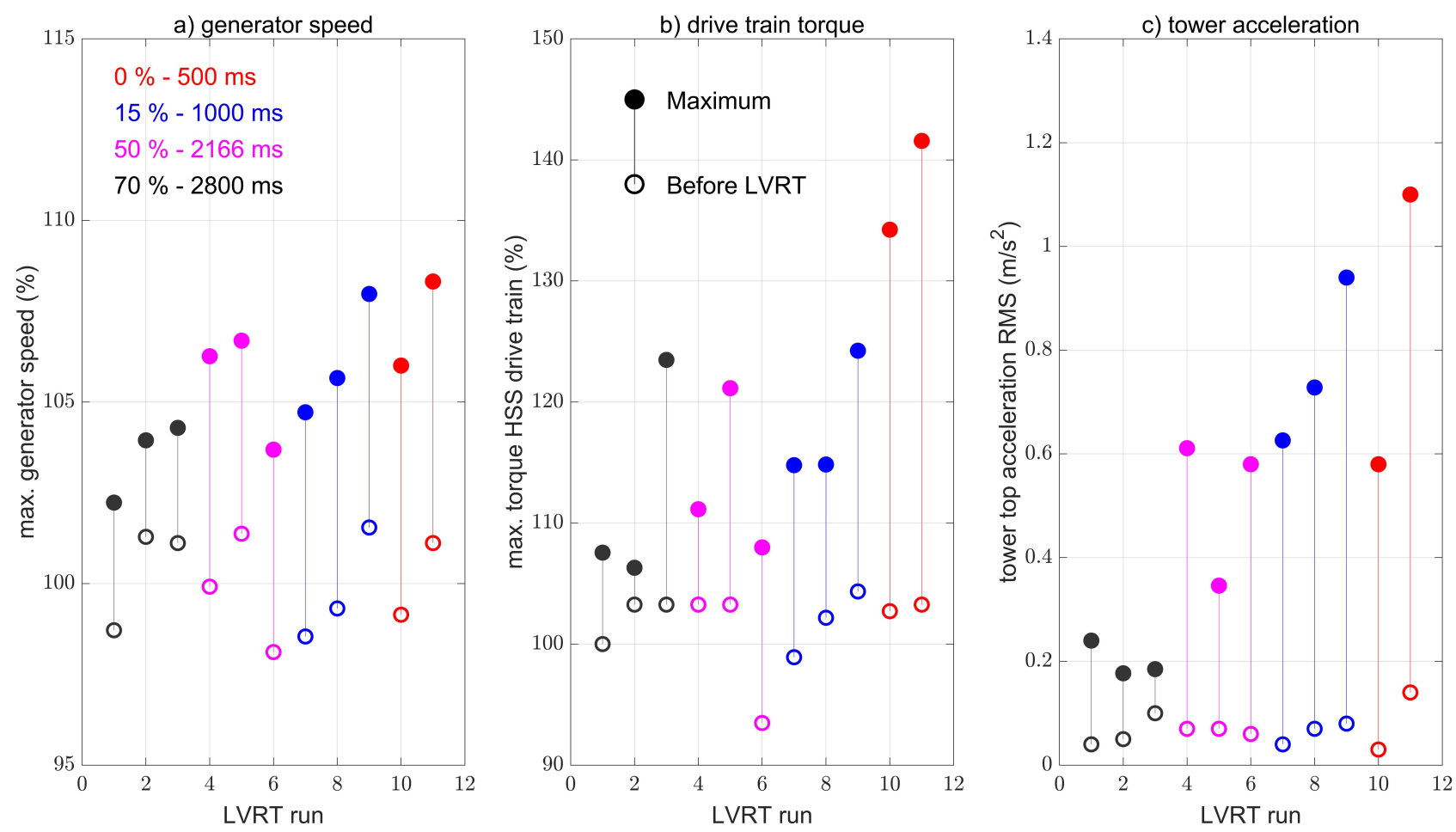

Figure 2. Measured values of generator speed (a), drivetrain torque (b), and tower acceleration (c) for 11 LVRT events of a measurement campaign. Tower top acceleration is given in absolute RMS value, whereas all other signals are relative to their nominal values. All events refer to voltage drops in all three current phases and at full power production. The color code corresponds to four different LVRT settings with varying voltage dip depth and duration, see the legend. Open circles refer to values right before the LVRT, whereas full circles refer to the maximum values during or after the LVRT.

\section{Rotor Acceleration during LVRT-Avoiding over Speed and Staying Connected}

To fulfill the grid requirement, it is essential that the turbine keeps connected to the grid and does not shut down during a low voltage event. In Section 2, it was clearly shown that the instant drop of electrical power leads to a sudden speed-up which could be harmful to the turbine.

To protect the turbine and its components, the speed range is limited, which determines the operational boundary conditions during an LVRT event.

Hence the major goal of turbine control during LVRT is to reduce the overall speed-up of the turbine, and, in particular, the drivetrain oscillations to a non-critical level below the turbine-specific trigger threshold for the rotational speed.

The maximum observed generator speed values for eleven selected LVRT events of a measurement campaign are displayed in Figure 2a. All scenarios refer to voltage drops in all three current phases and are performed at rated power. The four different classes of LVRT parameter sets are distinguished by color-coding. The following conclusion can be deduced:

1. The initial values right before the LVRT (open circles of the different LVRT events) are not constant but are spread around the rated point of speed operation due to the fluctuating wind speed. Already high initial speed values will be passed through the final maximum speed value during LVRT. Therefore, unfavorable wind gusts right before the LVRT can increase the criticality with regards to over-speed.

2. The increases of generator speed illustrated as distances between open and full circles indicate that deeper voltage dips lead to higher maximum rotational speeds. Only the two most severe scenarios of $15 \%$ for one second and $0 \%$ dip depth for $0.5 \mathrm{~s}$ show similar speed increases. This finding clearly shows that the rotor acceleration is affected by both voltage dip depth and duration of LVRT. 
3. LVRT events lead to a significant generator speed increase of up to $10 \%$, which is already challenging for the turbine to stay within its operational limits. The presented results in Figure 2 would show even higher values without appropriate intelligent control strategies which are applied on the investigated Suzlon wind turbine. It is worth mentioning that even the 0\% LVRT is manageable with DFIG based wind turbines.

To investigate in more detail the impact of voltage depth and duration on the acceleration of the drivetrain, we have performed a numerical simulation with the aero-elastic wind-turbine program Flex5 (Lyngby, Denmark) [16]. For rated wind speed, the simulations are evaluated with respect to the maximum occurred generator speed. Here, it is already considered that for lower voltage dip depth, the turbine has to stay connected for higher LVRT durations.

In Figure 3, these results are plotted dependent on the voltage dip depth and the LVRT duration, where the color, as well as the z-coordinate, refers to the maximum generator speed value for each combination.

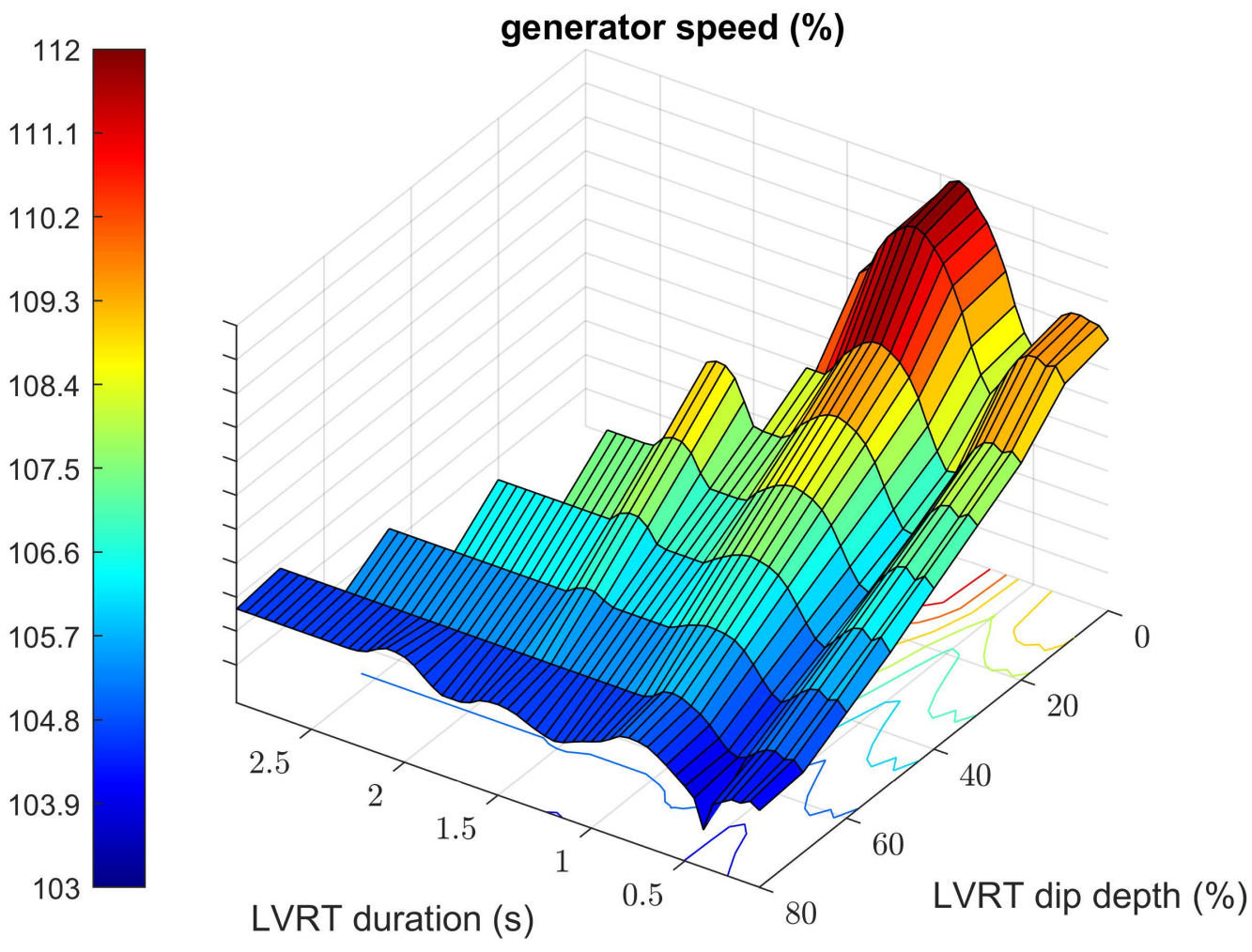

Figure 3. Simulation results of an LVRT parameter scan with Flex5. The maximum generator speed is plotted in dependency of the voltage dip depth and its duration. All values are normalized to the nominal generator speed, see color bar legend. Most critical parameter combinations lead to increases above $110 \%$.

Obviously, there is no strictly proportional trend between LVRT duration and the maximum final speed of the turbine. The results show a wave-like contribution of the maximum generator speed, which reflects the oscillatory behavior of the drivetrain during the LVRT event.

The findings are the following:

1. Overall, the maximum generator speed occurs with increasing duration and deeper voltage drop of the LVRT, see the highest speed values in the dark red color at the edge of lowest voltage and highest duration.

2. Additionally, the maximum occurring speed shows a wave-like distribution dependent on LVRT duration with a period time of roughly $0.6 \mathrm{~s}$ which corresponds in that 
simulation to the used eigenfrequency of $1.5 \mathrm{~Hz}$ of the drivetrain. The amplitude of these waves increases with deeper LVRT dip depth.

\section{LVRT Impact on Drivetrain Torque Loads}

The excitation of oscillation in the drivetrain is not only critical with regards to rotor speed but also from the loads' perspective. In the following section, we will show the different contributors to the overall drivetrain torque during an LVRT event. This helps in the following discussion of measured maximum torque values in the LVRT campaign from Figure 2.

Figure 4 shows the measured drivetrain torque during an LVRT scenario of a voltage dip depth of $50 \%$ and duration of $2 \mathrm{~s}$. Here the black line refers to the drivetrain torque measured with strain gauges at the high-speed shaft of the drivetrain.

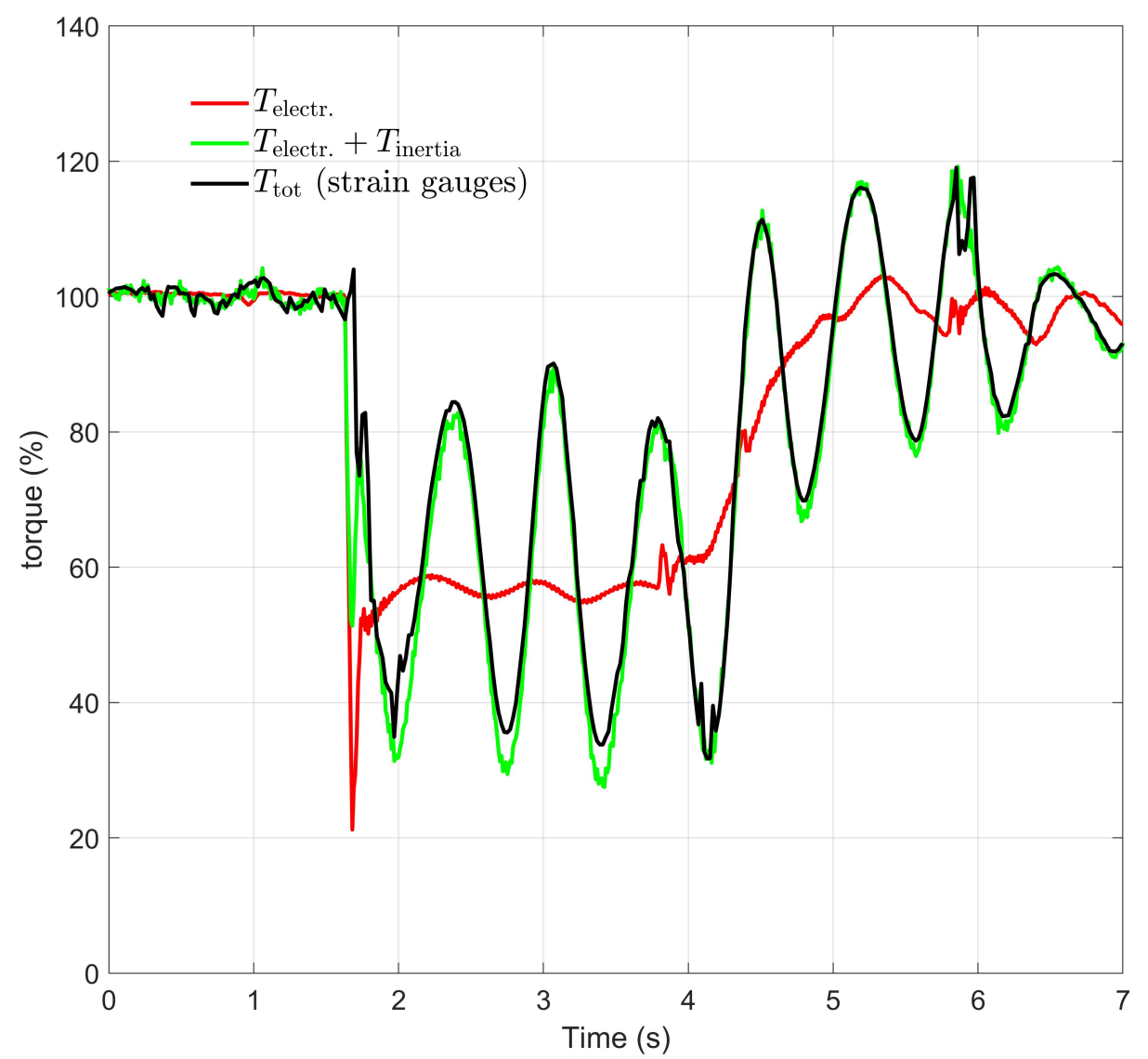

Figure 4. Measured drivetrain torque during an LVRT event with 50\% voltage dip depth and duration of two seconds. The black line corresponds to a signal measured by strain gauges on the high-speed shaft of the drivetrain. The green and red lines refer to calculated values that are derived from electric power and the rotational speed of the turbine.

As previously discussed, the sudden drop of electrical power induces strong oscillation in the drivetrain torque which persists for a long time due to low damping even when the voltage is back again. Therefore, the maximum observed torque occurs when the electrical power is fully back since, here, the oscillating torque $T_{\text {inertia }}$ superposes with the full air gap torque $T_{\text {electr }}$ of the generator.

$$
T_{\text {total }}=T_{\text {electr }}+T_{\text {inertia }}
$$

These two contributors to the torque can be well disentangled with two simple approaches based on the measured generated power and the rotational speed of the wind turbine. 
The air gap torque is illustrated by the red line and can be derived from the electrical power of the generator via:

$$
T_{\text {electr }}=P_{\text {electr }} / \omega
$$

which follows, in good approximation, the evolution of power.

The strong oscillations on top of the air gap torque can be well approximated with a simple model of a one-mass swing element which represents the components of the drivetrain. Its moment of inertia will contribute to the total torque load according to:

$$
T_{\text {inertia }}=\frac{d \omega}{d t} \cdot I_{H s s}
$$

where $I_{H S S}$ is the moment of inertia of the high-speed shaft drivetrain components, mainly the generator. The sum of both, air gap torque and the oscillatory part of the torque is represented by the green line in Figure 4 and is in good agreement with the strain gauge measured torque signal.

This example emphasizes the huge impact of the excited drivetrain oscillation on the overall maximum torque during and after an LVRT event.

For the various LVRT events, the maximum measured torque values are displayed in Figure 5, which relates to Figure $2 \mathrm{~b}$. The open circles again reflect the initial value right before the voltage drop and are spread near $100 \%$, which corresponds to the expected torque values at rated power production of the wind turbine.
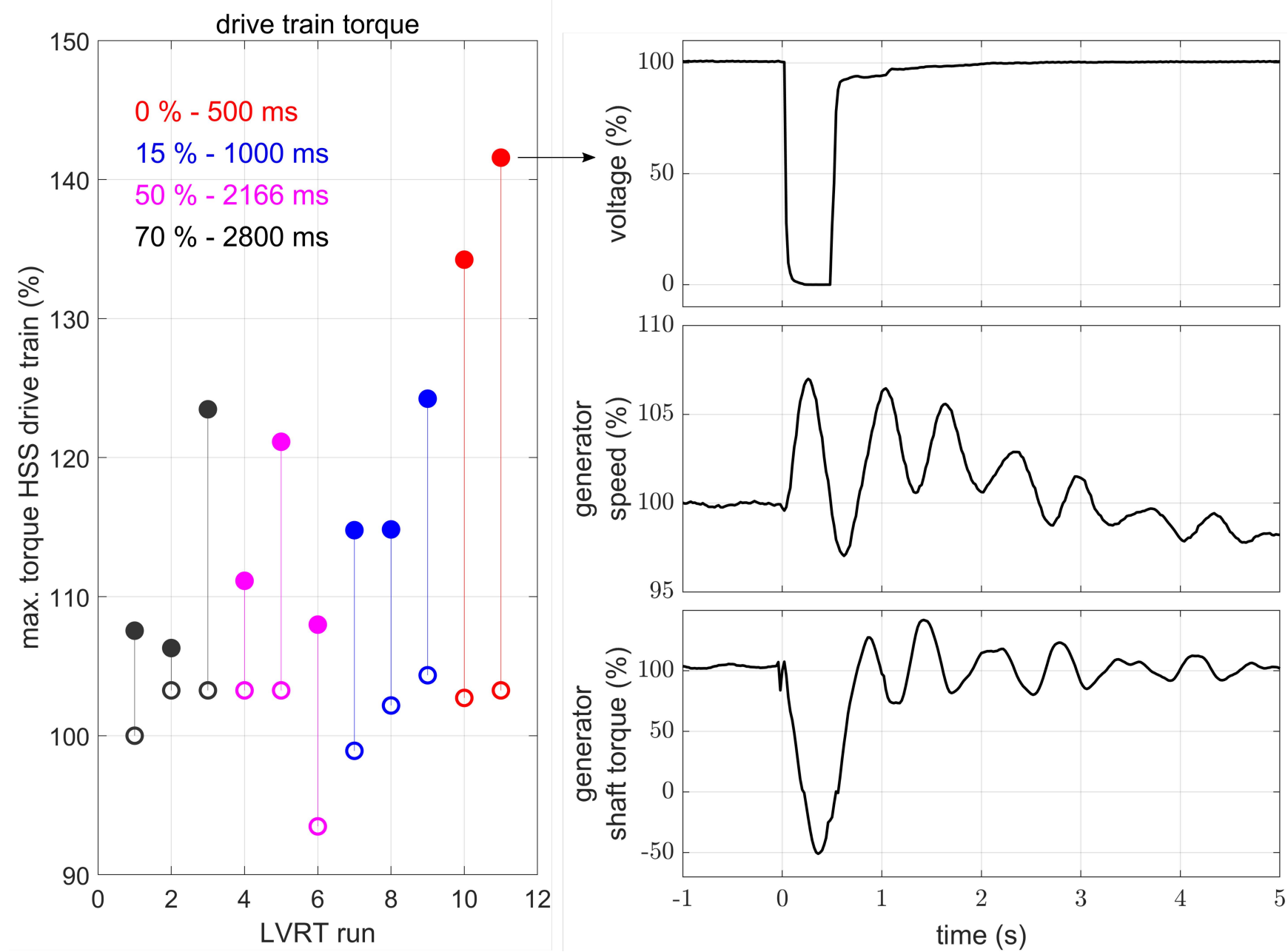

Figure 5. Overview of the measured drivetrain torque values for 11 LVRT events previously shown in Figure 2. The right inset shows the temporal evolution of the drivetrain torque, the generator speed, and the voltage for the most critical LVRT event with $0 \%$ voltage dip depth. All values are normalized to their nominal values. 
The full circles reflect the maximum torque values while or after LVRT. It is noticeable that the torque increase can differ strongly even for LVRT events with similar parameters, i.e., for second and third LVRT events of $70 \%$ voltage dip depth. This indicates that, especially for the less deep voltage drops, the maximum torque is not only affected by the power drop but also dependent on the wind situation and the actual power evolution during the sudden drop and sudden return of the voltage.

The maximum torque values of the majority of LVRT events are in the range of $105-125 \%$ of the nominal torque. The worst-case scenarios are clearly the $0 \%$-LVRTs, which show torque increases up to $140 \%$.

The right subplot of Figure 5 shows the time evolution of this worst-case $0 \%$-LVRT event to get more insight into the dynamical behavior of the drivetrain. It is striking that the immense power drop to zero leads to a sign change in the torque down to a value of $-50 \%$ whereas for a $15 \%$-LVRT event, the torque stays in the positive direction (not shown in the plot). This overshooting of torque leads to strong torque oscillation and therefore to the observed high maximum torque. This may bear the risk to be design driving for the main shaft and its components.

\section{Tower Loads and Tower Top Accelerations during LVRT Events}

The showcase example in Figure 1 of Section 2 has shown that LVRT events do not only lead to significant oscillations of the drivetrain but also impact the tower movement on a longer time scale. Here are the following two effects that contribute the most:

1. The sudden loss of aerodynamic rotor thrust due to the electrical power drop leads to tower bending motions in the fore-aft direction as well as to an impulse on the side-side motion.

2. The sudden increase of rotational speed will trigger the controller to pitch out immediately, where overshooting effects are likely to happen.

Since the pitch system and the tower reaction happen at a timescale of seconds, the highest amplitude of tower oscillation is reached some seconds after the LVRT, see Figure 1.

The tower top accelerations for different LVRT scenarios are shown in Figure 2c. Here a clear correlation between voltage dip depth and observed tower movement is visible. The highest accelerations of up to $1.2 \mathrm{~m} / \mathrm{s}^{2}$ are monitored for the deepest LVRT dip depths of $0 \%$ and $15 \%$.

In order to evaluate the severity of tower movement with respect to loads, we have measured additionally the tower bottom bending moments in the fore-aft direction. The results are plotted in Figure 6 for normal operation (Figure 6a) and execution of different LVRT scenarios (Figure 6b).

For both, Figure $6 \mathrm{a}, \mathrm{b}$, the load distribution of tower bottom bending moments in the fore-aft direction is plotted versus wind speed. The color indicates the number of recorded data points and therefore reflects some kind of load duration distribution dependent on wind speed.

Figure 6a shows data points of normal wind turbine operation, accumulated over a time of approximately one month for various wind conditions. Here the shape of the distribution clearly reflects the fact that the highest observed tower bending moments are observed at around rated wind speed of $9 \mathrm{~m} / \mathrm{s}$ where the rotor aerodynamics leads to the highest rotor thrust.

The same analysis of tower bending moments in the fore-aft direction is shown on Figure $6 \mathrm{~b}$ for the previously discussed LVRT events at various voltage dips and durations. Thus, the limited time of operation and henceforth the reduced number of data points lead to a different coloration, see color bar legend. However, the trend of maximum observed tower bending moments at rated wind speed is visible as well. 

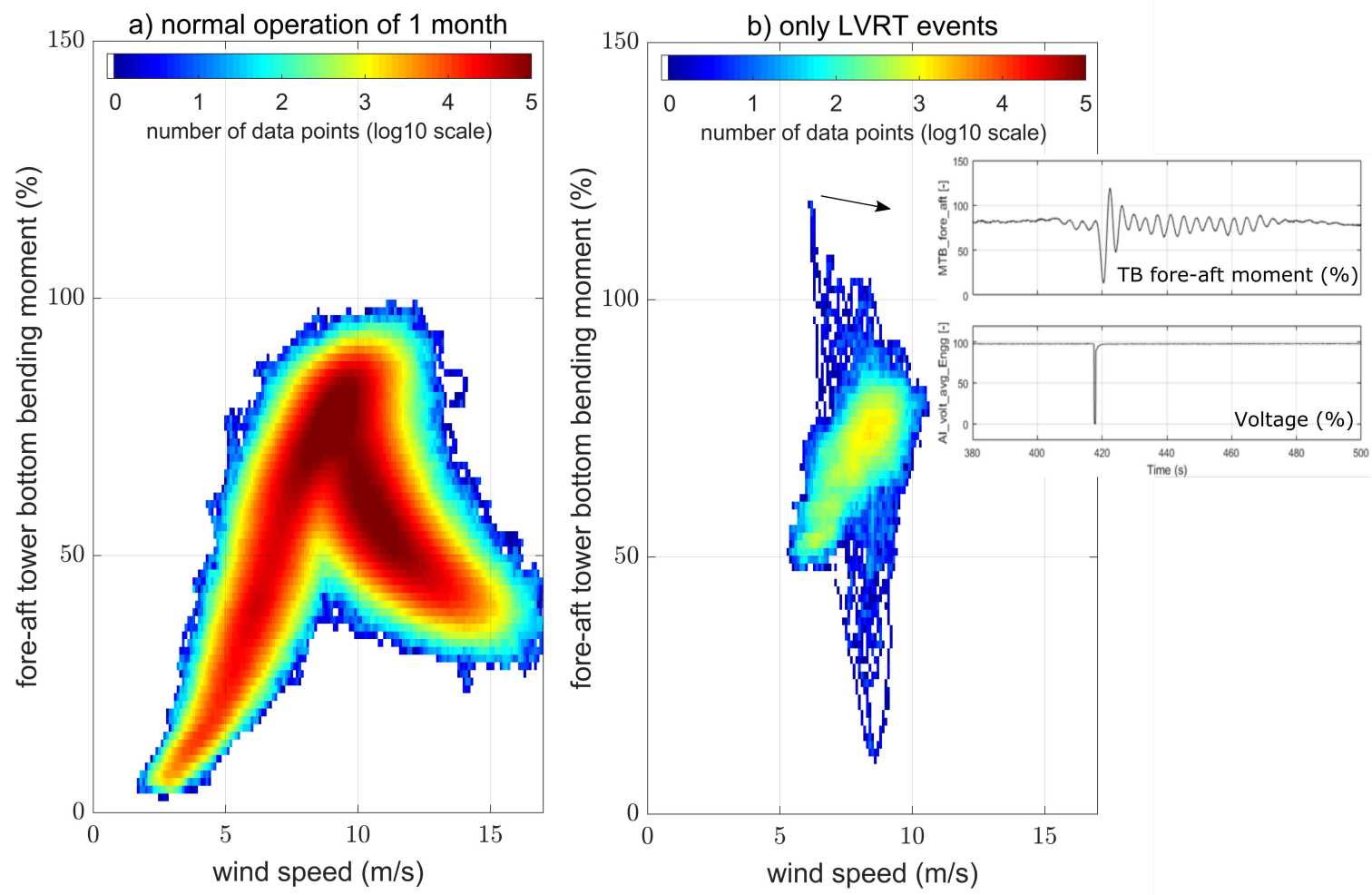

Figure 6. Distribution of measured tower bottom bending moments in fore-aft direction versus wind speed. The color code refers to the frequency of occurrence, i.e., the number of data points. Color coding is on a logarithmic scale in a way that even single data points appear in blue color. All values are normalized to the maximum observed value during normal operation. (a) shows normal wind turbine operation for a one-month period. The highest bending moments occur at rated wind speed near $10 \mathrm{~m} / \mathrm{s}$. (b) shows the same analysis for 11 LVRTs of a measurement campaign. Oscillations of tower moments during and after LVRT appear as traces in an up-down direction. Time evolution of the most critical LVRT is depicted in the inset panel.

In addition to the typical hook-shaped distribution from normal turbine operation, fine traces appear on top of this distribution towards higher and lower bending moments. These trails mark the evolution of tower bending moments during the variety of different performed LVRT events.

This wind speed-based comparison between LVRT driven tower loads (b) and expected loads during normal operation (a) enables meaningful conclusions about the impact of LVRT events on tower loads:

- It is clearly visible that most of the LVRT events have been performed near rated wind speed where the tower loads are expected to be the highest.

- Some traces of the LVRT events show load increases up to $125 \%$ depending on the specific LVRT scenario as well as on the wind conditions during the LVRT.

These results are not critical with respect to extreme load conditions for the tower. Design driving scenarios for the tower bottom extreme loads are typically idling scenarios during storm conditions or error scenarios with subsequent braking of the turbine. These extreme loads are typically twice as much as the loads during normal operation. Nevertheless, the significant acceleration of the tower during LVRTs may have to be considered in the turbine monitoring system to avoid false alarm turbine stoppage.

In addition to the observed tower bottom loads in a measured LVRT campaign, we performed LVRT simulations with Flex 5 to have a more detailed view of the sensitivity of tower vibration to the LVRT parameter dip depth and LVRT duration. Figure 7 displays, on the right side, the maximum observed tower top acceleration in the fore-aft direction 
in $\mathrm{m} / \mathrm{s}^{2}$, dependent on the LVRT duration and dip depth. In addition, three selected time series of $50 \%$ dip depth scenarios for different LVRT duration are shown on the left panel.

It is well visible that the tower movement is triggered by the fast pitch-out activity leading to the fore-aft tower bending against wind direction, see the deflection of the tower in the 4th row. For a longer LVRT duration, the turbine is pitching out for a longer time leading to a higher deflection within the first period of tower movement. Subsequently, the tower is moving back and starts oscillating with its eigenfrequency. When the power returns the turbine pitches back to its original value and pushes the tower in the wind direction. For the LVRT event of $600 \mathrm{~ms}$, red line, this pitch-in activity is in phase with the swing-back of the tower leading to higher overall tower acceleration compared to the LVRT event of $1200 \mathrm{~ms}$.

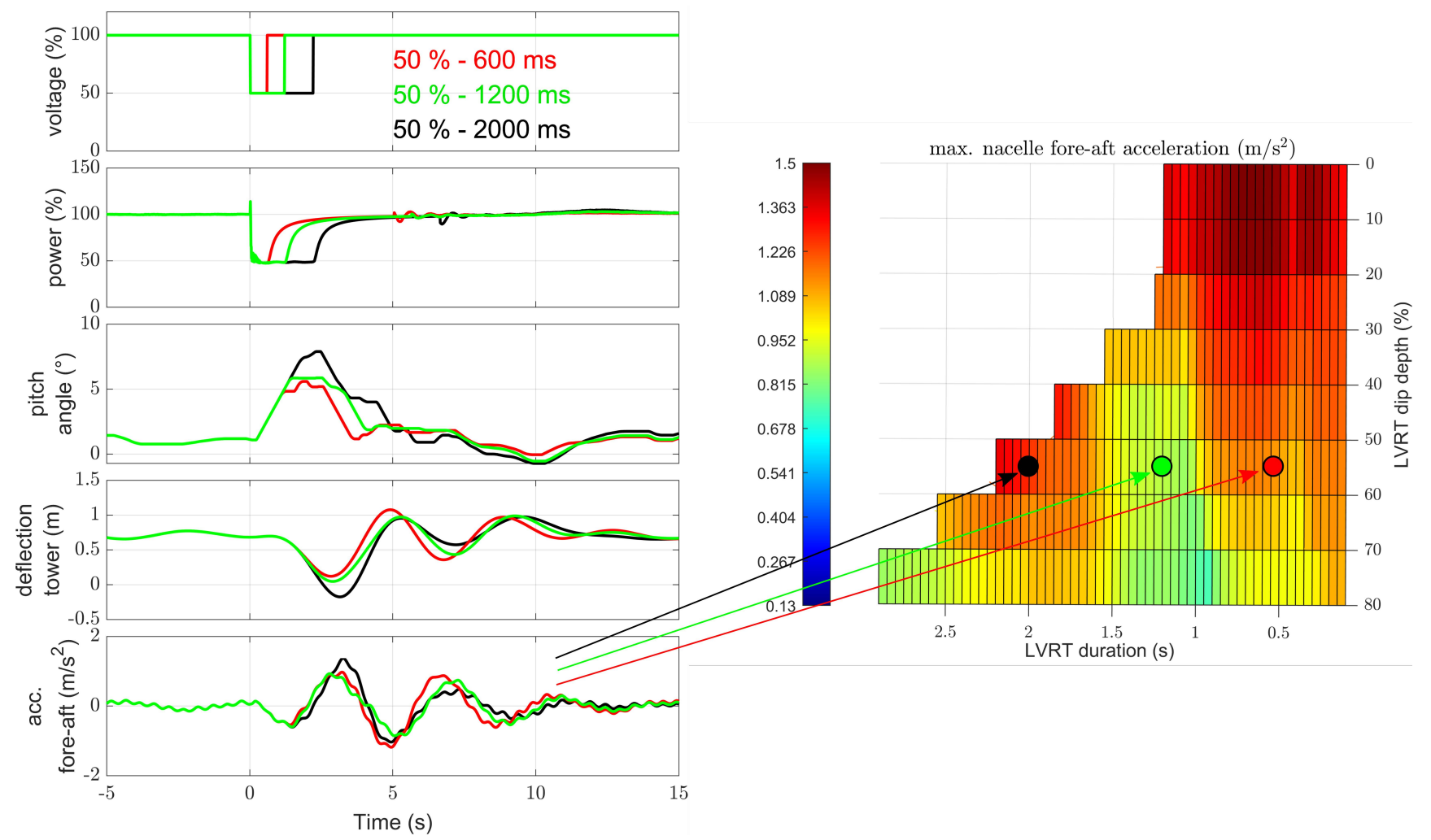

Figure 7. Flex5 simulation results for various combinations of LVRT duration and voltage dip depth at rated wind speed. The left panel shows the time series of three LVRT events at the same voltage dip depth of $50 \%$ and different LVRT duration, see the legend. The maximum observed tower acceleration in the fore-aft direction, see the last row, is illustrated in the right panel as a color-coded matrix for a parameter study of dip depth and duration.

This phase-dependency between pitch activity and tower movement is also visible in the false-color plot on the right side of Figure 7. Here the following conclusion can be drawn:

- Highest tower top accelerations are observed for the deepest voltage drops

- The return of voltage and the subsequent pitch activity has a significant influence on the final observed maximum tower acceleration. It appears that certain time windows are more critical, i.e., a twofold peak structure appears, which is in detail discussed in the corresponding time series to the left.

The simulation results confirm that LVRT events temporarily lead to significant tower vibrations with respect to normal operation but not to a level where they become critical for the integrity of the turbine. 


\section{Summary and Outlook}

Various LVRT events of different parameters have been carried out in an LVRT measurement campaign for Suzlon wind turbines in the $2 \mathrm{MW}$ class with rotor diameters larger than $100 \mathrm{~m}$ and a DFIG-based power system.

The measurement results clearly show that sudden drops of voltage are challenging scenarios for wind turbines and lead to significant impacts on mechanical loads.

The overall acceleration of the rotor in combination with significant oscillations in the drivetrain after the voltage drop can bring the turbine close or even beyond its operational limits, especially for long and deep LVRT events.

These drivetrain vibrations are also manifested in the torque signal, where the additional oscillating moment of inertia of the drivetrain lead to temporary values, which are up to $40 \%$ higher than the average torque during rated power production and may have to be considered in the design of the drivetrain components.

For the tower, it could be shown that LVRT events come with significant tower movements and accelerations up to $1.2 \mathrm{~m} / \mathrm{s}^{2}$. Simultaneous measurements of tower bottom bending moments exhibit loads that can be up to $125 \%$ higher than during normal operation but are still below values from typical design driving events.

All in all, it appears that the sudden increase in rotor speed and the temporary swingup of drivetrain torque push the turbine to its limits and are the most challenging problems to tackle.

Nevertheless, it was shown that even critical LVRT events with $0 \%$ voltage dip depth, duration of $0.5 \mathrm{~s}$, and power recovery to $90 \%$ within $1 \mathrm{~s}$ are still feasible with multiMegawatt DFIG-based wind turbines.

In the future, it is expected that new and stricter grid code requirements will further demand additional support from wind turbines. At the same time, the active response to grid frequency fluctuations and the response to multiple consecutive LVRT will stress the mechanical components of the turbine even further.

Author Contributions: Conceptualization, M.A., M.H. (Martin Hopp) and M.H. (Martin Huhn); software, M.H. (Martin Hopp) and M.A.; validation, M.A.; formal analysis, M.A. and M.H. (Martin Hopp); investigation, M.H. (Martin Hopp) and M.A.; writing-original draft preparation, M.A.; writing-review and editing, M.A., M.H. (Martin Hopp) and M.H. (Martin Huhn); visualization, M.A. and M.H. (Martin Hopp); funding acquisition, M.H. (Martin Hopp). All authors have read and agreed to the published version of the manuscript.

Funding: This research received no external funding.

Institutional Review Board Statement: Not applicable.

Informed Consent Statement: Not applicable.

Data Availability Statement: 3rd Party Data-Restrictions apply to the availability of these data.

Acknowledgments: We acknowledge financial support by Land Schleswig-Holstein within the funding program Open Access Publikationsfonds. We further acknowledge Suzlon Energy Limited for the ability to publish the presented results.

Conflicts of Interest: The authors declare no conflict of interest.

\section{References}

1. Central Electricity Authority. Notification 6th February 2019; No.12/X/STD(CONN)/GM/CEA/2018; Central Electricity Authority: New Delhi, India, 2018.

2. International Electrotechnical Commission (IEC). Wind Energy Generation Systems. Part 1: Design Requirements; IEC 61400-1 Edition 4; IEC: Geneva, Switzerland, 2019.

3. Fraunhofer Institute for Energy Economics and Energy System Technology (IEE). Annual Turbine Installations by Turbine Concept. Available online: http://windmonitor.iee.fraunhofer.de/windmonitor_en/3_Onshore/2_technik/3_antriebskonzepte/ (accessed on 29 April 2021). 
4. Rini, A.J.; Prabaharan, N.; Palanisamy, K.; Umashankar, S.; Thirumoorthy, A. A Review on Low Voltage Ride Through capability in wind turbines of India and challenges in implementation. In Proceedings of the 1st International Conference on Large-Scale Grid Integration of Renewable Energy, New Delhi, India, 6-8 September 2017.

5. Li, B.; Liu, J.; Wang, X.; Zhao, L. Fault Studies and Distance Protection of Transmission Lines Connected to DFIG-Based Wind Farms. Appl. Sci. 2018, 8, 562. [CrossRef]

6. Pidikiti, T.; Tulai, R.D.G. Analysis and Performance Evaluation of DFIG and PMSG Based Wind Energy Systems. Int. J. Comput. Digit. Syst. 2019, 8. [CrossRef]

7. Hiremath, R.; Moger, T. Comprehensive review on low voltage ride through capability of wind turbine generators. Int. Trans. Electr. Energ. Syst. 2020, 30, e12524. [CrossRef]

8. Qin, B.; Li, H.; Zhou, X.; Li, J.; Liu, W. Low-Voltage Ride-Through Techniques in DFIG-Based Wind Turbines: A Review. Appl. Sci. 2020, 10, 2154. [CrossRef]

9. Tohidi, S.; Behnam, M.-i. A comprehensive review of low voltage ride through of doubly fed induction wind generators. Renew. Sustain. Energy Rev. 2016, 57, 412-419. [CrossRef]

10. Yang, L.; Xu, Z.; Ostergaard, J.; Dong, Z.Y.; Wong, K.P. Advanced Control Strategy of DFIG Wind Turbines for Power System Fault Ride Through. IEEE Trans. Power Syst. 2012, 27, 713-722. [CrossRef]

11. Naderi, S.B.; Davari, P.; Zhou, D.; Negnevitsky, M.; Blaabjerg, F. A Review on Fault Current Limiting Devices to Enhance the Fault Ride-Through Capability of the Doubly-Fed Induction Generator Based Wind Turbine. Appl. Sci. 2018, 8, 2059. [CrossRef]

12. Wessels, C.; Fuchs, F.W. Fault ride through of DFIG wind turbines during symmetrical voltage dip with crowbar or stator current feedback solution. In Proceedings of the 2010 IEEE Energy Conversion Congress and Exposition, Atlanta, GA, USA, 12-16 September 2010; pp. 2771-2777. [CrossRef]

13. Miao, F.; Shi, H.; Zhang, X. Impact of the Converter Control Strategies on the Drive Train of Wind Turbine during Voltage Dips. Energies 2015, 8, 11452-11469. [CrossRef]

14. Wenske, J.; Beckert, U. Voltage-induced stresses during Low Voltage Ride Through (LVRT) in the drive train of wind turbines with DFIG. In Proceedings of the International Conference on Renewable Energies and Power Quality (Icrepq'12), Santiago de Compostela, Spain, 28-30 March 2012.

15. Huhn, M.; Hopp, M.; Jauch, C. Additional Design Criteria for Wind Turbines: Excitation to Mechanical Vibrations from the Power System. In Proceedings of the 6. VDI Fachtagung Schwingungen von Windenergieanlagen, Bremen, Germany, 16-17 June 2015; Verein Deutscher Ingenieure (VDI): Dusseldorf, Germany, 2015.

16. Passon, P.; Kühn, M.; Butterfield, S.; Jonkman, J.; Camp, T.; Larsen, T.J. OC 3 -Benchmark exercise of aero-elastic offshore wind turbine codes. J. Phys. Conf. Ser. 2007, 75, 12. [CrossRef]

17. Wessels, C.; Fuchs, F.W. LVRT of DFIG wind turbines-Crowbar vs. stator current feedback solution. In Proceedings of the EPE Wind Energy Chapter Symposium 2010, Staffordshire University, Stafford, UK, 15-16 April 2010. 